\title{
Stability of Quantum Fluids: Wavy Interface Effect
}

\author{
A. Kwang-Hua CHU [*] \\ P.O. Box 39, Tou-Di-Ban, Road XiHong, Urumqi 830000, PR China
}

\begin{abstract}
A numerical investigation for the stability of the incompressible slip flow of normal quantum fluids (above the critical phase transition temperature) inside a microslab where surface acoustic waves propagate along the walls is presented. Governing equations and associated slip velocity and wavy interface boundary conditions for the flow of normal fluids confined between elastic wavy interfaces are obtained. The numerical approach is an extension (with a complex matrix pre-conditioning) of the spectral method. We found that the critical Reynolds number $\left(R e_{c r}\right.$ or the critical velocity) decreases significantly once the slip velocity and wavy interface effects are present and the latter is dominated $\left(R e_{c r}\right.$ mainly depends on the wavy interfaces).

PACS numbers : 68.03.-g, 67.57.Np, 67.57.Hi, 67.55.Hc, 67.40.Vs, 43.35.Lq
\end{abstract}

\section{Introduction}

Ultrasound measurements play very important roles in a ${ }^{3} \mathrm{He}$ investigation ${ }^{1}$. The time scale of ultrasound corresponds to quasiparticle life times at low temperatures and first to zero sound crossover was observed. Energy scales of ultrasound match the binding energies of Cooper pairs in superfluid ${ }^{3} \mathrm{He}$ were observed coupling through density oscillations. Sound transmission method using many types of surface acoustic wave (SAW) sensors have been developed for acoustical and electrical property measurements of adjacent liquid or gas (especially liquid ${ }^{4} \mathrm{He}$ at low temperature ${ }^{2}$ ). A Rayleigh-SAW propagates along the substrate surface by emitting compressional waves into the quantum fluid and thus the sampling of the Rayleigh-SAW is determined by the acoustic impedance of the surrounding quantum fluids. Relevant problems are the instability for the flow induced by the $\mathrm{SAW}^{3,4}$ or the slip effect existing along the interface of the quantum fluid. In fact, the instability may triggers the laminar-turbulent transition for flows of the superfluid. The important issue is to determine the critical velocity ${ }^{5,6}$ for the relevant physical parameters so that the experimental measurements could be under control.

A most striking characteristic of liquid helium is that it exists in the liquid state down to the absolute zero temperature because (i) the van der Waals forces in helium are weak; (ii) the zeropoint energy, due to the light mass, is large. In fact, it is called a quantum liquid due to these kinds of quantum effects and is closely related to the Bose-Einstein condensation for He II. The well-known properties of He II can be largely accounted for on the basis of phenomenological two-fluid theory ${ }^{7,8}$. One of the basic assumptions of the two-fluid model is : He II consists of a kind of mixture of two components, a normal component and a superfluid component. The 
former has viscosity while the superfluid can move without friction as long as certain velocity limits are not exceeded.

One crucial issue for the related researches about He II is the critical velocity ${ }^{5,6,9}$ (when it flows through a capillary or plane channel) which depends on the micro-channel size. Landau attributed the existence of a critical velocity in He II to the breakdown of the superfluid due to the creation of excitations (he proposed that phonons and rotons are two types of excitations which make up the normal fluid). If the velocity is less than the critical value, there will be no dissipation or friction along the boundary or interface for the flow. In plane channel or slab flows of quantum fluids, to determine the critical velocity corresponds to finding out the critical Reynolds number ${ }^{5-6,10}$.

The traditional starting point of an investigation of hydrodynamic stability is eigenvalue analysis, which proceeds in : (i) linearize about the laminar solution and then (ii) look for unstable eigenvalues of the linearized problem. In much of the literature of hydrodynamic stability ${ }^{10}$, attention has been restricted to 2D perturbations, in particular, the well-known Orr-Sommerfeld equation is an eigenvalue equation for $2 \mathrm{D}$ perturbations. For pressure-driven flow, with the no-slip boundary conditions (which are normally valid in macro-channels), eigenvalue analysis ${ }^{11}$ predicts a critical Reynolds number $R e_{c r}=5772$ at which instability should first occur, but in the laboratory ${ }^{12}$, transition to turbulence is observed at $R e_{c r}$ as low as $\sim 1000$.

As for quantum fluids or liquids, there always exists a slip velocity along the static interface or confinement due to the microscopically incomplete momentum exchange therein ${ }^{13}$. The distinction among various flow regimes can be obtained by introducing the Knudsen number (which also characterizes the value of the slip velocity13), $\mathrm{Kn}=\mathrm{mfp} / L$, where $\mathrm{mfp}$ is the mean free path and $L$ is the characteristic flow dimension. Slip flow conditions exist for $0.001<\mathrm{Kn} \leq 0.1$, where the flow can be considered continuous if the slip velocity at the walls are taken into account. Meanwhile, the effect of elastic or deformable interfaces, like surface acoustic waves (SAW) interacted with volume and surface phonons (propagating along the elastic boundaries ${ }^{14-17}$ ), upon the stability of quantum fluid flows, however, were seldom considered before, to the best knowledge of the authors. Although the mathematical difficulty is essential therein. Note that, entrainment of fluids induced by SAW propagating along deformable boundaries have been studied $^{14}$ since early 1950s. The role of elastic macroscopic walls resembles that of microscopic phonons ${ }^{4}$. As presented in [4], for the description of the transport processes in the nonequilibrium gas-solid system including the processes occurring in the case of propagation of sound in a solid, we need to solve

$$
\begin{aligned}
& \frac{\partial f(\mathbf{x}, \mathbf{v}, t)}{\partial t}+\mathbf{v} \cdot \frac{\partial f(\mathbf{x}, \mathbf{v}, t)}{\partial \mathbf{x}}=I_{g}(\{f\}), \\
& \frac{\partial n\left(\mathbf{x}, \mathbf{k}_{j}, t\right)}{\partial t}+\mathbf{c}_{j} \cdot \frac{\partial n\left(\mathbf{x}, \mathbf{k}_{j}, t\right)}{\partial \mathbf{x}}=I_{v}(\{n\}), \\
& \frac{\partial H\left(\mathbf{r}, \mathbf{K}_{\xi}, t\right)}{\partial t}+\mathbf{c}_{\xi} \cdot \frac{\partial H\left(\mathbf{r}, \mathbf{K}_{\xi}, t\right)}{\partial \mathbf{r}}=I_{s}(\{f, n, H\}),
\end{aligned}
$$


and the associated boundary conditions (scattering and interacting laws near the interface)

$$
\begin{aligned}
& \left|v_{r}\right| f^{+}=\int_{v_{i}<0} d \mathbf{v}_{i}\left|v_{i}\right| f^{-}\left(\mathbf{v}_{i}\right) W\left(\mathbf{v}_{i} \rightarrow \mathbf{v}_{r}\right), \\
& \frac{\left|c_{r}\right|}{L_{t}} n^{-}\left(\mathbf{k}_{j}\right)=\sum_{\mathbf{k}_{j_{1}}\left(c_{i}>0\right)}\left[\frac{c_{i}}{L_{t}} n^{+}\left(\mathbf{k}_{j_{1}}\right)+\bar{N}_{g}\left(\mathbf{k}_{j_{1}}\right)+\bar{N}_{p}\left(\mathbf{k}_{j_{1}}\right)\right] V_{p}\left(\mathbf{k}_{j_{1}} \rightarrow \mathbf{k}_{j} ; \omega\right) .
\end{aligned}
$$

$f, n$, and $H$ denote the distribution function for gases, volume phonons, and surface phonons, respectively. $I_{g}, I_{v}$, and $I_{s}$ are the corresponding collision integrals. Please see the details in [4] for other notations or symbols. The stability problems for such a system of differential-integral equations remain open up to now. To escape from above (many-body problems) difficulties, we plan to use the macroscopic approach which is a complicated extension of previous approaches ${ }^{16,18}$. In this work, the stability analysis of incompressible slip flows for a normal fluid (above the transition temperature $T_{c}$ ) inside a microchannel with the flow confined by two elastic (wall) layers of thin films where surface (acoustic) waves are propagating along the interfaces is conducted. Namely, we will relax the static- or rigid-interface boundary conditions which are frequently used in previous theoretical and/or experiment approaches into the dynamic- or deformable-interface boundary conditions which are more realistic in essence when we consider the flow stability problem in a microdomain where surface acoustic waves ${ }^{15}$ propagating along the boundaries of quantum fluids ${ }^{2,4}$. The verified code which was based on the spectral method developed by $\mathrm{Chu}^{18}$ will be extended here to include the boundary conditions coming from SAW existing along the interfaces to obtain the stability characteristics of the basic flow.

This paper is organized as follows. We introduce the mathematical formulation for the OrrSommerfeld equation and the relevant linear stability analysis in Section 2. Boundary conditions for the slip flow and the wavy interface of the fluid system are then incorporated. The expression for primary slip flows of quantum fluids we shall investigate their stability characteristic will be derived before we describe the numerical approach : a modified spectral method at the end of this Section. Two physical parameters, $K_{0}$ (relevant to the SAW effect) and Kn will be introduced or defined. Finally we shall present our calculations and discuss them. Our results illustrate that the critical Reynolds number $\left(R e_{c r}\right)$ decreases (to 1441 ) rapidly once the interfaces are subjected to propagating waves (or noises) and there are slip velocities existing along the boundaries or interfaces $\left(K_{0}=1\right.$ and $\left.K n=0.001\right)$. However, the slip velocity (adjusted by $\mathrm{Kn}$ ) effect is minor and adverse compared to the SAW effect (tuned by $K_{0}$ ) considering the decrease of $R e_{c r}$.

\section{Governing Equations}

Macroscopically, the motion of the normal fluid (above the critical phase transition temperature of the quantum fluids) as a whole could be treated by using hydrodynamical models starting 
from the microscopic atomic wave function ${ }^{8,19}$. Here, after the simplifying treatment of the complicated mathematical derivations, the dimensionless equations of motion for an incompressible normal fluid flow $8,10,11,18$, in the absence of body forces and moments, reduce to

$$
\frac{\partial \mathbf{U}}{\partial t}+(\mathbf{U} \cdot \nabla) \mathbf{U}=-\nabla P+\frac{1}{R e} \nabla^{2} \mathbf{U}
$$

where $\mathbf{U}$ and $P$ stand for the velocity and pressure of fluids. $R e=\rho u_{\max } h / \mu$ is the Reynolds number with $\rho$ and $\mu$ being the density and viscosity of fluids. For the case of normal fluid flows driven by a constant pressure-gradient, i.e., plane Poiseuille flow, the length scale is the half width of the normal-fluid layer $h$, and the velocity is the center-line velocity $u_{\max }$. Following the usual assumptions of linearized stability theory ${ }^{10-11}, U_{i}\left(x_{i}, t\right)=\bar{u}_{i}\left(x_{i}\right)+u_{i}^{\prime}\left(x_{i}, t\right)$, and similarly, $P\left(x_{i}, t\right)=\bar{p}\left(x_{i}\right)+p^{\prime}\left(x_{i}, t\right)$, the linearized equation, which governs the disturbances are:

$$
\frac{\partial u_{i}^{\prime}}{\partial t}+(\overline{\mathbf{u}} \cdot \nabla) u_{i}^{\prime}+\left(\mathbf{u}^{\prime} \cdot \nabla\right) \bar{u}_{i}=-\nabla p^{\prime}+\frac{1}{R e} \nabla^{2} u_{i}^{\prime}
$$

Disregarding the lateral disturbances, $w^{\prime}=0$, a stream function for the disturbance, $\psi$, can be defined such that $u^{\prime}=\partial \psi / \partial y, v^{\prime}=-\partial \psi / \partial x$. Using normal mode decomposition analysis, $\psi$ may be assumed to have the form $\psi(x, y, t)=\phi(y) \exp [i \alpha(x-C t)], \alpha$ is the wave number (real), $C$ is $C_{r}+i C_{i}$. This is a kind of Tollmien-Schlichting transversal waves, $C_{r}$ is the ratio between the velocity of propagation of the wave of perturbation and the characteristic velocity, $C_{i}$ is called the amplification factor, and $\alpha$ equals to $2 \pi \Lambda^{-1}$, where $\Lambda$ is the wave length of the TollmienSchlichting perturbation ${ }^{10}$. Substituting the stream function and eliminating the pressure, we have the linearized disturbance equation

$$
\left(D^{2}-\alpha^{2}\right)\left(D^{2}-\alpha^{2}\right) \phi=i \alpha \operatorname{Re}\left[(\bar{u}-C)\left(D^{2}-\alpha^{2}\right) \phi-\left(D^{2} \bar{u}\right) \phi\right]
$$

where $D=d / d y$. This is also valid for the slip flow regime ${ }^{13}, 0.001<K_{n} \leq 0.1$, since the flow can still be considered as continuous.

\subsection{Boundary Conditions}

For the slip flow, the continuous models can be used if the no-slip boundary condition is modified. A few models have been suggested to estimate the nonzero velocity at a boundary surface ${ }^{13,16}$. In this study, we adopt the approach based on Taylor's expansion of the velocity around the wall. Thus, the first order approximation yields $\left.\bar{u}\right|_{\text {wall }}=\mathrm{Kn} d \bar{u} / d y$ (positive for the inner normal as $y \equiv n)$. Consequently, the mean (basic) velocity profile is given by

$$
\bar{u}=1-y^{2}+2 \mathrm{Kn}
$$

for $-1 \leq y \leq 1$. Boundary conditions for $\phi$ or $D \phi$ are not the same as previous no-slip approach, i.e., $\phi( \pm 1)=D \phi( \pm 1)=0$ and shall be introduced below. 


\subsection{Interface Treatment}

We consider a two-dimensional layer (slab) of uniform thickness filled with a homogeneous normal fluid (Newtonian viscous fluid; its dynamics is described by Navier-Stokes equations). The upper and lower boundaries of the layer are superfluids which are rather flexible, on which are imposed travelling sinusoidal waves of small amplitude $a$ (due to SAW or peristaltic waves). The vertical displacements of the upper and lower interfaces $(y=h$ and $-h)$ are thus presumed to be $\eta$ and $-\eta$, respectively, where $\eta=a \cos \frac{2 \pi}{\lambda}(x-c t), \lambda$ is the wave length, and $c$ the wave speed. $x$ and $y$ are Cartesian coordinates, with $x$ measured in the direction of wave propagation and $y$ measured in the direction normal to the mean position of the interfaces. It would be expedient to simplify these equations by introducing dimensionless variables. We have a characteristic velocity $c$ and three characteristic lengths $a, \lambda$, and $h$. The following variables based on $c$ and $h$ could thus be introduced :

$$
x^{\prime}=\frac{x}{h}, \quad y^{\prime}=\frac{y}{h}, \quad u^{\prime}=\frac{u}{c}, \quad v^{\prime}=\frac{v}{c}, \quad \eta^{\prime}=\frac{\eta}{h}, \quad \psi^{\prime}=\frac{\psi}{c h}, \quad t^{\prime}=\frac{c t}{h}, \quad p^{\prime}=\frac{p}{\rho c^{2}},
$$

where $\psi$ is the dimensional stream function. The amplitude ratio $\epsilon$, the wave number $\alpha$, and the Reynolds number $R e_{c}$ are defined by

$$
\epsilon=\frac{a}{h}, \quad \alpha=\frac{2 \pi h}{\lambda}, \quad R e_{c}=\frac{c h}{\nu} .
$$

After introducing the dimensionless variables, now, for the ease and direct representation of our mathematical expressions in the following, we shall drop those primes (') in those dimensionless variables and treat them as dimensionless. We seek a solution in the form of a series in the parameter $\epsilon$ :

$$
\psi=\psi_{0}+\epsilon \psi_{1}+\epsilon^{2} \psi_{2}+\cdots, \quad \frac{\partial p}{\partial x}=\left(\frac{\partial p}{\partial x}\right)_{0}+\epsilon\left(\frac{\partial p}{\partial x}\right)_{1}+\epsilon^{2}\left(\frac{\partial p}{\partial x}\right)_{2}+\cdots,
$$

with $u=\partial \psi / \partial y, v=-\partial \psi / \partial x$. The $2 \mathrm{D}$ ( $\mathrm{x}-$ and $\mathrm{y}-$ ) momentum equations and the equation of continuity for the normal fluid could be in terms of the stream function $\psi$ if the pressure $(p)$ term is eliminated. The final governing equation is

$$
\frac{\partial}{\partial t} \nabla^{2} \psi+\psi_{y} \nabla^{2} \psi_{x}-\psi_{x} \nabla^{2} \psi_{y}=\frac{1}{R e_{c}} \nabla^{4} \psi, \quad \nabla^{2} \equiv \frac{\partial^{2}}{\partial x^{2}}+\frac{\partial^{2}}{\partial y^{2}},
$$

and subscripts indicate the partial differentiation. If we presume originally the fluid is quiescent; this corresponds to a free pumping case and finally the velocity profile of the fluid is symmetric with respect to the centerline of the plane channel bounded by the superfluids. Equation above, together with the condition of symmetry and a uniform constant pressure-gradient in the $\mathrm{x}$ direction, $(\partial p / \partial x)_{0}=$ constant, yield :

$$
\psi_{0}=K_{0}\left[(1+2 \mathrm{Kn}) y-\frac{y^{3}}{3}\right], \quad K_{0}=\frac{R e_{c}}{2}\left(-\frac{\partial p}{\partial x}\right)_{0},
$$

$K_{0}$ is in fact a necessary pumping to sustain a plane Poiseuille flow (pressure-driven case). $\psi_{0}$ corresponds to the solution of

$$
\frac{\partial}{\partial t} \nabla^{2} \psi_{0}+\psi_{0 y} \nabla^{2} \psi_{0 x}-\psi_{0 x} \nabla^{2} \psi_{0 y}=\frac{1}{R e_{c}} \nabla^{4} \psi_{0}
$$


and

$$
\psi_{1}=\frac{1}{2}\left\{\phi(y) e^{i \alpha(x-t)}+\phi^{*}(y) e^{-i \alpha(x-t)}\right\},
$$

where the asterisk denotes the complex conjugate. The normal fluid is subjected to boundary conditions imposed by the symmetric motion of the wavy interfaces and the slip condition at interfaces. The basic slip flow now has this form (cf [16]), as $u=\partial \psi_{0} / \partial y$,

$$
\bar{u}=1-y^{2}+2 \mathrm{Kn},
$$

where $c$ is the phase speed of the $\mathrm{SAW}, \mathrm{Kn}=\mathrm{mfp} / h$. Boundary conditions become

$$
\phi_{y}( \pm 1) \pm \phi_{y y}( \pm 1) \mathrm{Kn}=2 K_{0}(1 \pm \mathrm{Kn}), \quad \phi( \pm 1)= \pm 1 .
$$

\subsection{Numerical Approach}

The eigenvalue problem raised above could be solved by using the verified $\operatorname{code}^{18}$, which used the spectral method ${ }^{20}$ based on the Chebyshev-polynomial-expansion approach, once the equation and boundary conditions are discretized. For instance, we have, from equation (3), as a finitesum approximation (reduction from $\infty$ to $\mathrm{N}$ ),

$$
\phi(z)=\sum_{n=0}^{N} a_{n} T_{n}(z)
$$

where $T_{n}(z)$ is the Chebyshev polynomial ${ }^{11,20}$ of degree $n$ with $z=\cos (\theta) . T_{n}(z)$ are known to satisfy the recurrence relations

$$
z T_{n}(z)=\frac{1}{2}\left[T_{n+1}(z)+T_{n-1}(z)\right]
$$

After substituting $\phi$ into (3) and with tremendous manipulations, we obtain the algebraic equation

$$
\begin{gathered}
\frac{1}{24} \sum_{\substack{p=n+4 \\
p \equiv n(\bmod 2)}}^{N}\left[p^{3}\left(p^{2}-4\right)^{2}-3 n^{2} p^{5}+3 n^{4} p^{3}-p n^{2}\left(n^{2}-4\right)^{2}\right] a_{p}- \\
\sum_{\substack{p=n+2 \\
p \equiv n(\bmod 2)}}^{N}\left\{\left[2 \alpha^{2}+\frac{1}{4} i \alpha \operatorname{Re}\left(4 M_{0} f-4 C-M_{0} c_{n}-M_{0} c_{n-1}\right)\right] p\left(p^{2}-n^{2}\right)-\frac{1}{4} i \alpha \operatorname{Re} M_{0} c_{n} p\left[p^{2}-(n+2)^{2}\right]-\right. \\
\left.\frac{1}{4} i \alpha \operatorname{Re} M_{0} d_{n-2} p\left[p^{2}-(n-2)^{2}\right]\right\} a_{p}+i \alpha \operatorname{Re} M_{0} n(n-1) a_{n}+\left\{\alpha^{4}+i \alpha \operatorname{Re}\left[\left(M_{0} f-C\right) \alpha^{2}-2 M_{0}\right]\right\} c_{n} a_{n}- \\
\frac{1}{4} i \alpha^{3} \operatorname{Re} M_{0}\left[c_{n-2} a_{n-2}+c_{n}\left(c_{n}+c_{n-1}\right) a_{n}+c_{n} a_{n+2}\right]=0
\end{gathered}
$$

for $n \geq 0, f=1+2 K_{n}$, where $c_{n}=0$ if $n>0$, and $d_{n}=0$ if $n<0, d_{n}=1$ if $\mathrm{n} \geq 0$. Here, $M_{0}=1, C$ is the complex eigenvalue. The boundary conditions become

$$
\sum_{\substack{n=1 \\ n \equiv 1(\bmod 2)}}^{N} a_{n}=1, \quad \sum_{\substack{n=1 \\ n \equiv 1(\bmod 2)}}^{N}\left[n^{2}+\operatorname{Kn} \frac{n^{2}\left(n^{2}-1\right)}{3}\right] a_{n}=2 K_{0} .
$$


The matrices thus formed are of poor condition because they are not diagonal, symmetric ${ }^{21}$. Thus, before we perform floating-point computations to get the complex eigenvalues, we precondition these complex matrices to get less errors. Here we adapt Osborne's algorithm to precondition these complex matrices via rescaling, i.e., by certain diagonal similarity transformations of the matrix (errors are in terms of the Euclidean norm of the matrix) designed to reduce its norm. The details of this algorithm could be traced in [18,21-22]. The form of the reduced matrix is upper Hessenberg. Finally we perform the stabilized $L R$ transformations for these matrices to get the complex eigenvalues (please see also $[18,22]$ for the details). The preliminary verified results of this numerical code had been done for the cases of $\mathrm{Kn}=0$ (no-slip boundary conditions) in comparison with the bench-mark results of Orszag's ${ }^{11}$. For example, for $R e=10000.0, \alpha=1.0$ of the test case : plane Poiseuille flow, we obtained the same spectra as $0.23752648+$ i 0.00373967 for $C_{r}+$ i $C_{i}$ which Orszag obtained in 1971.

\section{Results and Discussion}

After careful verification, we proceed to obtain (through tremendous searching using doubleprecision machine accuracy) the detailed spectra for the illustration of the stability of the slip flow in normal fluids confined between wavy interfaces. To demonstrate some of the calculated spectra $\left(C_{r}, C_{i}\right)$ near the regime of $\left(R e_{c r}, \alpha\right)$, we plot Fig. 1 by selecting 2 pairs of $(R e, \alpha)=(1562,1.156)$ and $(2982.3,1.0783)$ with the corresponding $K_{0}=1$, and 0.5 for the same Knudsen number $(\mathrm{Kn}=0.01)$. Once $C_{i}>0$, the instability occurs! The onset of instability is easy to occur once the Reynolds number, wave number, or Knudsen number perturb a little again near this regime $\left(C_{i}\right.$ becomes zero and then positive).

We then plot the neutral stability boundary curves for different cases in Fig. 2. It is clear that each curve is composed of two branches (one is upper and the other is lower, and they coalesce into a critical point $\left(R e_{c r}\right.$ and $\left.\alpha_{c r}\right)$ as the Reynolds number decreases). We tune the $K_{0}$ parameter to be 1 and 0.5, with the corresponding Knudsen number being 0.001 and 0.01, respectively. Once the Knudsen number is set to be zero and there is no SAW effect, we recover the curve obtained by $\operatorname{Orszag}^{11}\left(R e_{c r} \sim 5772\right)$. Otherwise, the resulting critical Reynolds numbers $\left(R e_{c r}\right)$ are 1441,1562, 2664, 2982.3, respectively. It seems the effect of SAW propagating along the interface is the dominated one and will degrade the flow stability significantly. The slip velocity effect is minor and adverse (delay the transition).

To understand the stability behavior related to the decay or amplification of the perturbed disturbance waves in the finite time for certain mode, we also illustrate their time evolution patterns by selecting the least unstable mode. As illustrated in Figs. 3 and 4 for Re=1441, $\alpha=1.175\left(K_{0}=1\right)$ and $\operatorname{Re}=2664, \alpha=1.105\left(K_{0}=0.5\right)$, we can observe the oscillating or amplifying pattern just after a finite time (time is dimensionless and the Knudsen number is the same, $\mathrm{Kn}=0.001$ ). The original disturbance (wave) will not decay for these unstable modes 
$\left(C_{r}, C_{i}\right) \sim(0.382,0.000002)$, and $\sim(0.324,0.00000046)$, respectively.

We can finally conclude that various kinds of interface noises (as illustraed here, slip velocities and the propagating surface acoustic wavs) will premature any instability mechanism considering the temporal growth of the disturbances. We have obtained more clues about the slip flow (which is in a non-equilibrium state) instability of quantum fluids (above their critical transition temperature) by considering more realistic interface conditions. Once we know the viscosities and/or densities of these quantum fluids, based on the obtained critical Reynolds number, we can then determine the critical velocity ${ }^{5,6}$ for each case. Meanwhile, these results will help researchers to understand the formation or generation of vorticity waves and then the route to low-temperature turbulence in quantum fluids. It seems the range of wave numbers relevant to the SAW propagating along the flexible interfaces, the Knudsen numbers and the Reynolds

numbers of basic slip flows of normal fluids must be carefully selected for the optimal flow control usage in SAW applications ${ }^{1,2}$ to the investigation of ${ }^{3} \mathrm{He}$. Our further study will be relevant to those more complicated issues ${ }^{2-3,22-25}$.

Acknowledgements. The author is partially supported by the National Natural Science Foundation of China (NNSFC) under grant No. : 10274061 and the China Post-Dr. Science Foundation (grant No. :1999-17).

\section{References}

[*] The author is from : P.O. Box 30-15, Shanghai 200030, PR China. Address before Septemper 2004 : Department of Basic Science, Qinghai University, Cheng-Bei District, XiNing 810016, PR China.

[1] W.P. Halperin and E. Varoquaux, in : W.P. Halperin and L.P. Pitaevskii (eds.), Helium 3 (North-Holland, Amsterdam, 1990).

[2] Y. Aoki, et al, Physica B 329-333, 234 (2003). K. Dransfeld and E. Salzmann, in : Physical Acosutics, (eds.) W.P. Mason, R.N. Thurston (Academic Press, New York, 1970).

[3] R.L. Willett, et al, Phys. Rev. Lett. 65, 112 (1990). P. Terry and M.W.P. Strandberg, J. Appl. Phys. 52, 4281 (1981).

[4] V.D. Borman, S.Yu. Krylov, and A.M. Kharitonov, Sov. Phys. JETP 65, 935 (1987).

[5] V.P. Peshkov, in : Proc. VII Int'l. Conf. Low Temp. Phys., Toronto, p. 555 (1960).

[6] W.F. Vinen, Physica B 329-333, 191 (2003). R.J. Donnelly, Physica B 329-333, 1 (2003). W. Jr. Zimmermann, Contemp. Phys. 37, 219 (1996).

[7] L. Tisza, Phys. Rev. 72, 838 (1947). 
[8] L.D. Landau and E.M. Lifshitz, Fluid Mechanics (Pergamon Press, 2nd. ed., New York, 1987).

[9] J. Wilks, Rep. Prog. Phys. 20, 38 (1957). A.D.B. Woods and R.A. Cowley, Rep. Prog. Phys. 36, 1135 (1973).

[10] P.G. Drazin and W.H. Reid, Hydrodynamic Stability (Cambridge University Press, London, 1981). W. Heisenberg, Ann. Physik (Leipzig) 74, 577 (1924).

[11] S.A. Orszag, J. Fluid Mech. 50, 689 (1971).

[12] J.K. Platten and J.C. Legros, Convection in Liquids (Springer, Berlin, 1984).

[13] D. Einzel and J.M. Parpia, J. Low Temp. Phys. 109, 1 (1997). A. Kundt and E. Warbung, Pogg. Ann. Phys. 156, 177 (1875).

[14] M.S. Longuet-Higgins, Philos. Trans. R. Soc. London 345, 535 (1953).

[15] R.M. Moroney, R.M. White, and R.T. Howe, Appl. Phys. Lett. 59, 774 (1991).

[16] A. K.-H. Chu, Electronics Lett. 38, 1481 (2002).

[17] S. Mizuno, Physica B 316-317, 230 (2002). P. Král and M. Shapiro, Phys. Rev. Lett. 86, 131 (2001). A. Sergeev and V. Mitin, Physica B 316-317, 276 (2002). E. Varoquaux, et al, Physica B 178, 309 (1992).

[18] A. K.-H. Chu, IEE Proc. Nanobiotechnol. 150, 21 (2003). K.-H. Chu and C.-C. Chang, in Proceedings of National Aeronautics and Astronautics Conf. (AASRC, Taipei/Taiwan; Dec. 1990) pp. 199.

[19] R.P. Feynman, R.P., Phys. Rev. 94262 (1954). I.M. Khalatnikov and V.V. Lebedev, J. Low temp. Phys. 32, 789 (1978). N.B. Kopnin, Rep. Prog. Phys. 65, 1633 (2002).

[20] D. Gottlieb and S.A. Orszag, Numerical Analysis of Spectral Methods : Theory and Applications (NSF-CBMS Monograph No. 26, SIAM, 1977).

[21] J. H. Wilkinson, The Algebraic Eigenvalue Problem (Oxford University Press, 1965). E.E. Osborne, J. Assoc. Comput. Mach. 7, 338 (1960).

[22] A. K.-H. Chu, Preprint (2002).

[23] G. Gervais, et al, Phys. Rev. Lett. 88, 045505 (2002).

[24] A.J. Legget, Rev. Mod. Phys. 71, S318 (1999). R.J. Donnelly, J. Phys. Condens. Matter 11, 7783 (1999). D.C. Samuels, Phys. Rev. B 46, 11714 (1992).

[25] T. Nomura, A. Saitoh, and S. Furukawa, in : Proc. 1999 IEEE Ultrasonics Symp., 1999, p. 477. A.P. Mayer, Phys. Rep. 256, 237 (1995). 


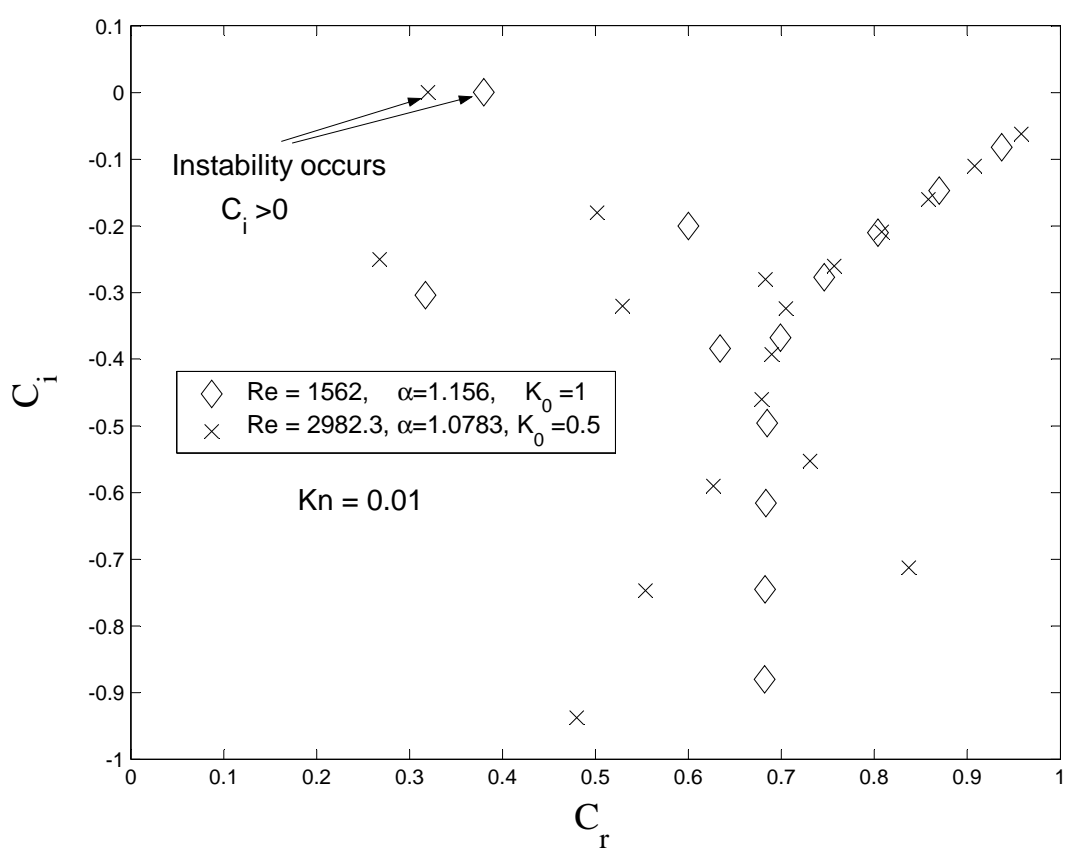

Fig. 2 Illustration of the temporal spectra $\left(C_{r}, C_{i}\right)$ for disturbance waves due to interface $\left(K_{0}\right)$ and slip velocity $(\mathrm{Kn}=0.01)$ effects.

$R e=1562,2982.3$ for corresponding $K_{0}=1,0.5$ and $\alpha=1.156,1.0783$, respectively.

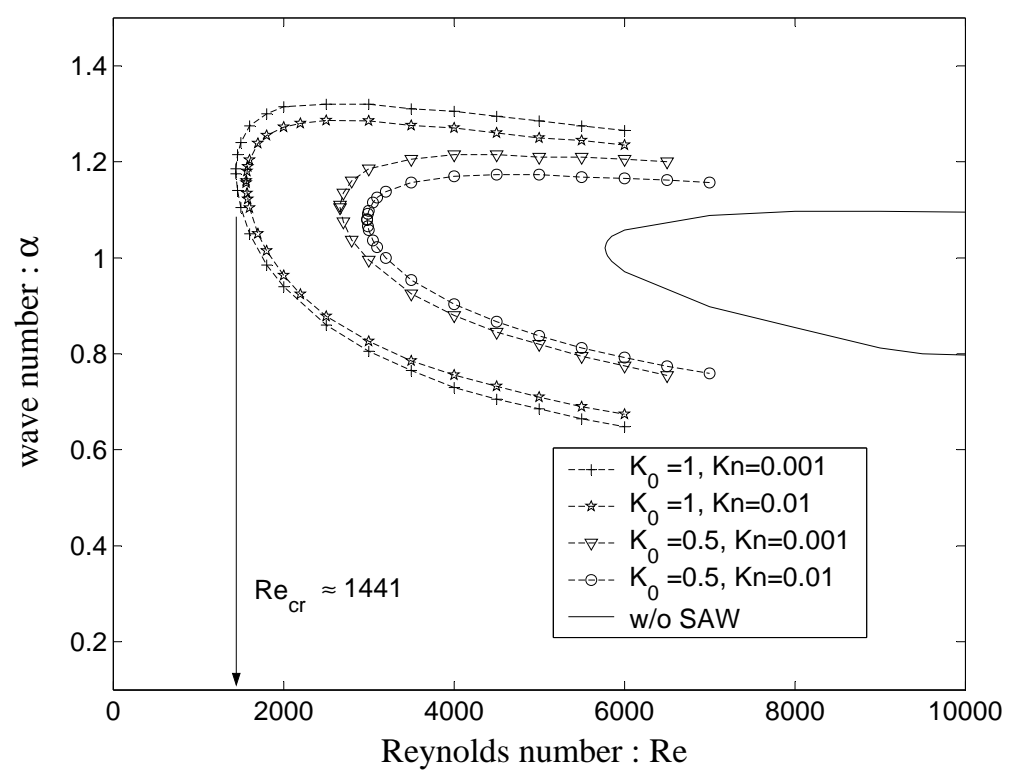

Fig. 3 Comparison of wavy interface $\left(K_{0}\right)$ and slip velocity $(\mathrm{Kn})$ effects on the neutral stability boundary of the basic flow. $\mathrm{Kn}=\mathrm{mfp} / h . \mathrm{mfp}$ is the mean free path of the quantum fluid. $R e_{c r} \sim 1441,1562,2664,2982.3$ for $K_{0}=1: \mathrm{Kn}=0.001,0.01$, and $K_{0}=0.5: \mathrm{Kn}=0.001,0.01$. 


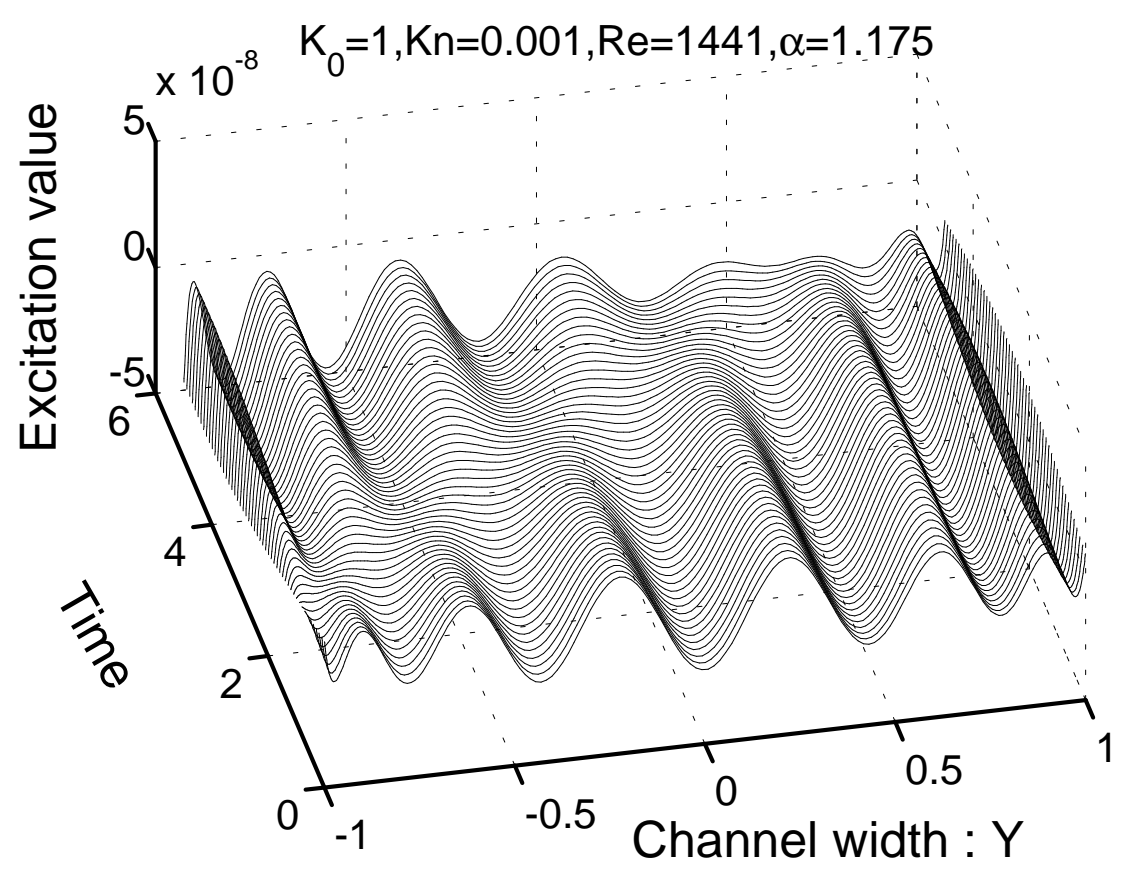

Fig. 3 Illustration of the temporal evolution for disturbance waves due to interface $\left(K_{0}\right)$ and slip velocity $(\mathrm{Kn})$ effects.

$R e=1441$ for corresponding $K_{0}=1$ and $\alpha=1.175, \mathrm{Kn}=0.001$. Time is dimensionless.

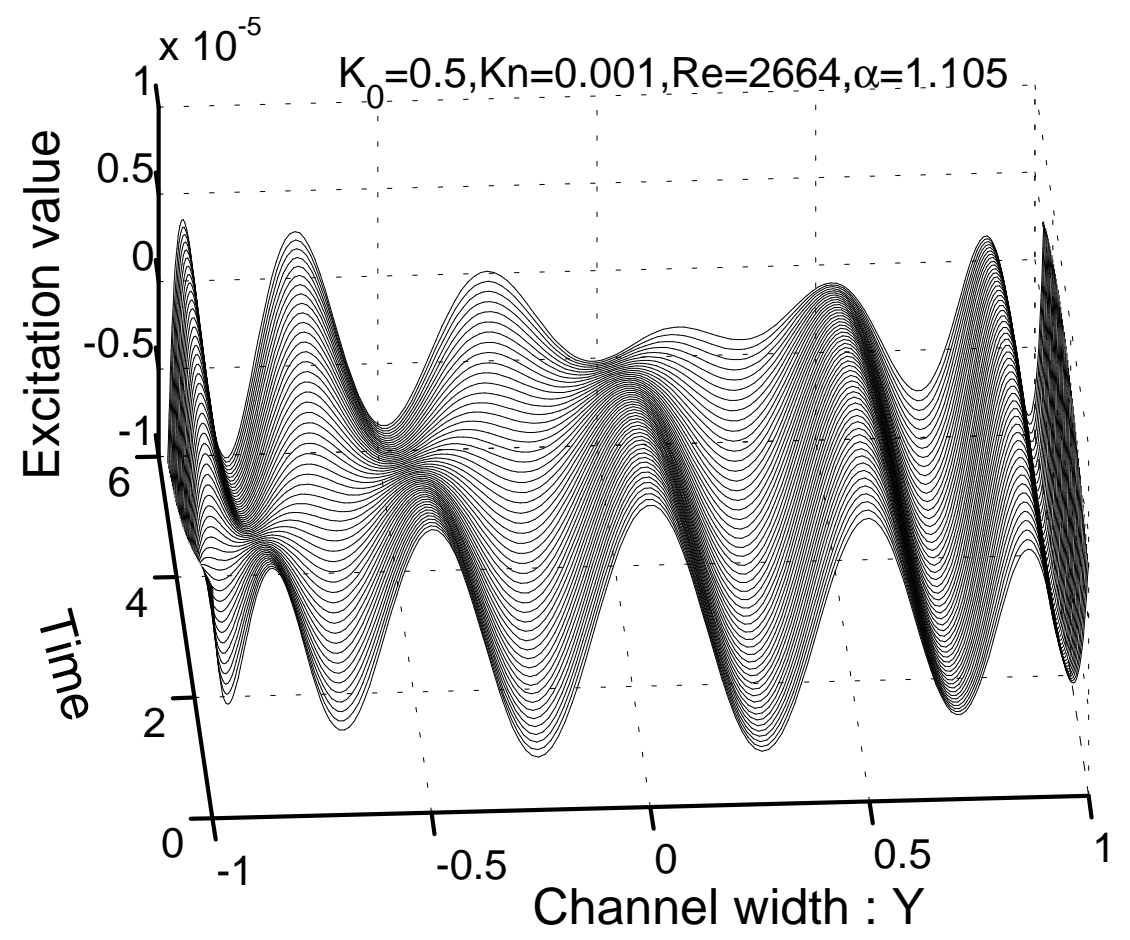

Fig. 4 Illustration of the temporal evolution for disturbance waves due to interface $\left(K_{0}\right)$ and slip velocity $(\mathrm{Kn})$ effects.

$R e=2664$ for corresponding $K_{0}=0.5$ and $\alpha=1.105, \mathrm{Kn}=0.001$. Time is dimensionless. 